\title{
Use of Cathodoluminescence Scanning Electron Microscope with Color TV Display for Study of Natural Diamonds Internal Structure
}

Saparin, G.V. ${ }^{1}$, Obyden S.K. ${ }^{1}$, and Titkov, S. V. ${ }^{2}$

1. Physical Department, Moscow State University, Vorobiovy gory, 119899, Russia

2. Institute of Geology of Ore Deposits, Petrography, Mineralogy and Geochemistry, Russian Academy of Sciences, Staromonetny per. 35, Moscow 109017, Russia

\section{Introduction}

For a study of natural diamonds internal structure, such methods as birefringence and Schlieren micrography, X-ray diffraction topography, the etching of surface, ultraviolet absorption, as well as photo- and cathodoluminescence topography have been applied. Cathodoluminescence $(\mathrm{CL})$ topography became recently one of the most frequently employed technique. To obtain CL-pattern it is usually utilized defocused electron beam flooding the diamond plate surface, which is optically imaged on photographic film. In this report advantages of application of focused electron beam operating in scanning mode in electron microscope (SEM) with color TV-display of video information are consern.

\section{Technique description}

We offer a new developed version CL-technique nondistorted by relief influence and color CL-SEM images with composite black/wite and color contrasts (Obyden et al. 1997) which is based on the CL-system described earlier (Saparin and Obyden, 1988). In this case the SEM synthesises an image presenting independent color CL contrast and microtopography simultaneously. This technique is more flexible technique for CL-topography investigation uses the SEM operating in CL-mode and especially in color CL (CCL) - mode as well as backscattered electrons (BSE)-mode forming black/white component of contrast. The $(\mathrm{CCL}+\mathrm{BSE})-\mathrm{SEM}$ technique was designed for the localization of the space distribution of trace impurity concentration and structural defects in luminescent media. It offers a highly sensitive technique for qualitative and quantitative luminescence analysis of materials and devices with high spatial resolution (about $0.2-0.5 \mu \mathrm{m}$ ). The image on the monitor screen is formed by real colors (not color coding!) in accordance with the local CL-spectrum for each 
pixel in wave length interval $400-800 \mathrm{~nm}$. This color contrast technique is a unique way for quick presentation of CL spectra distribution over specimen surface ( in particular $512 \times 512$ pixels $\times 32 \mathrm{bit} /$ pixel $\times 6 \mathrm{~s}$ ). Above mentioned method is contactless technique and allows to study of physical and optical properties of the materials without destruction of the specimen under investigation. The sensitivity of $\mathrm{CL}$ method to impurities is characterized by the detection limit of the concentration about $10^{14} \mathrm{~cm}^{-3}$. (CCL+BSE) image has an information capacity in over 100 times more than black/white CL-images . Apart high resolution capability, the technique provide wide range of magnification, from $5^{x}$ to $100 \mathrm{~K}$, permiting study both common view of sample surface and certain fine-scale details of interest. Unfortunately, for the study of bulk crystal internal structure by this technique it is necessary to cut the plates from the crystrals (or select cut stone), because the Cl-emission is exited by $20 \mathrm{kV}$ electrons only from the surface layers about $3 \mu \mathrm{m}$ in thickness.

\section{Results}

For analysis of growth defects in detail, set of 30 plates cut from natural diamonds from Yakutian deposits (Russia) were selected for low level of long-range elastic strains and postgrowth deformation defects. These strains and deformation defects, which were occured in the most of natural diamonds during their transportation from the Mantle to the Earth surface by kemberlites, interfere with study of the growth defects.

Application of CL topography to investigation of diamond plates revealed that a lot of natural crystals grew on micro-diamond seeds. Some seeds appear to be of irregular round form, another ones are characterized by crystallographic form with very complex banding. Sometimes growing together of two seeds was observed.

It was shown, that usually diamond crystals exibit very complex growth stratigraphy. Flat as well as curving growth layers was observed. In octahedral sectors it were fixed both flat and curving layers, but in cubic sectors only curving ones. Within some crystals an alternation of flat and curving bands was detected.

In accordance with previous work ( Lang, 1979 ), it was established that the common feature of growth sector configuration of most diamonds is original mixed development of (111) and (100) forms with growth out of the later at the final stage. Nevertheless, much more complex cases were discovered. In particular, in one crystal inspected, a growth history was 
disclosed as following: round seed $\Rightarrow$ cube $\Rightarrow$ octahedron $\Rightarrow$ cubo-octahedron $\Rightarrow$ octahedron. This sequences reflect the evolution of growth conditions.

It is possible to suggest, that the study of representative collection of diamond crystals may provide more complete data on processes of their nucleation and growth within Upper Mantle depths. Comparison of internal structures of natural and synthetic stones may be utilized as a criterion for their identification.

\section{References}

Lang, A.R., 1979, Internal structure: in J.E.Field (ed.), The properties of Diamond: Academic Press, London, Chap. 14, p. 425.

Obyden, S.K. , Ivannikov, P.V. , Saparin, G. V, 1997, Color Cathodoluminescence Display in the Scanning Electron Microscope of Deep Relief Surfaces: Scanning, v.19, p. 533-540.

Saparin, G.V. and Obyden,S.K, 1988, Color display of videoinformation in scanning electron microscopy: Principles and applications to physics, geology, soil scinces, biology and medicine: Scanning, 10, 87-106. 